\title{
Does acupuncture the day of embryo transfer affect the clinical pregnancy rate? Systematic review and meta-analysis
}

\author{
Juan-Enrique Schwarze ${ }^{1,2}$, Juan Pablo Ceroni ${ }^{2}$, Carolina Ortega-Hrepich ${ }^{3}$, Sonia Villa ${ }^{3}$, Javier Crosby ${ }^{1}$, Ricardo \\ Pommer ${ }^{3}$ \\ ${ }^{1}$ Unidad de Medicina Reproductiva Clinica Las Condes, Santiago, Chile \\ ²Departamento OB/GIN, Universidad de Santiago, Santiago, Chile \\ 3Unidad de Medicina Reproductiva Clinica Monteblanco, Santiago, Chile
}

\begin{abstract}
The effects of acupuncture on IVF outcomes is still unknown. We carried out a systematic review and meta-analysis of RCT to determine whether acupuncture performed at the time of ET improves outcomes. We searched Medline and Embase from January 1990 to June 2017, for the following terms): (acupuncture; acupuncture therapy) and (reproductive techniques, assisted; in vitro fertilization; embryo transfer). We selected RCT that compared acupuncture with sham acupuncture or no treatment. We included only trials in which acupuncture involved the insertion of needles into traditional meridian points. We evaluated the methodological quality of the trials using the Cochrane risk of bias tool. The measure of treatment effect was the pooled odds ratio of achieving a clinical pregnancy, ongoing pregnancy, or live birth for women in the acupuncture group compared with women in the control group. For pooled data, summary test statistics were calculated using the Mantel-Haenszel method, using the Rev-Man software, version 5.1. We analyzed six studies, including 2,376. In all trials, there were no significant differences between the groups concerning the mean numbers of embryos transferred, the mean age of the women undergoing the procedure, diagnose and use of ICSI. Acupuncture performed the day of ET was associated with a reduced risk of clinical pregnancy $(0.87,95 \%$ confidence interval 0.77 to 0.98$)$. The pooled rate difference was $-0.06(-0.12$ to -0.01$)$ for clinical pregnancy. None of the trials reported significant adverse effects of acupuncture.
\end{abstract}

Keywords: acupuncture, IVF, meta-analysis

\section{INTRODUCTION}

Approximately $10 \%$ of couples in reproductive age suffer from infertility. For many of these couples, in vitro fertilization with embryo transfer (IVF-ET) provides the best possibility to achieve pregnancy. However, only $20 \%$ of initiated cycles and $35 \%$ of embryo transfers result in the delivery of a healthy baby (Zegers-Hochschild et al., 2016a;b). Therefore, repeated treatment cycles are needed to achieve pregnancy. The need for repetition, not only place significant economic pressure on the couple, but it is also invasive and time-consuming.

After achieving blastocyst development, implantation is the factor with the greatest limitation on IVF-ET. Governed by complex mechanisms, the interaction between embryo and endometrium depends on the quality of each. New therapies that can improve this process are highly desirable.
As an important part of traditional Chinese medicine, acupuncture has gained increased popularity worldwide due to its convenience, lack of side effects, and unique therapeutic effects. Acupuncture has been used in China for centuries to regulate the female reproductive system (Chang et al., 2002). As a method of treating disease, the theory of acupuncture is based on the energy flow of qi and the principles of traditional Chinese medicine meridians and acupoints. It is said that the imbalance of qi in the body causes disease, which can be treated by stimulating specific acupoints on the body surface.

Some potential mechanisms for its effects on fertility have been postulated (Chang et al., 2002). Acupuncture may stimulate blood flow to the uterus by inhibiting uterine central sympathetic nerve activity (Stener-Victorin et al., 1996), and may stimulate the production of endogenous opioids, which may inhibit the central nervous system outflow and the biological stress response (Yang et al., 2008).

However, the effect of acupuncture on IVF outcomes is still unknown. Since the first relevant clinical research was published in 1999 a growing number of studies have been performed to explore the therapeutic effects of acupuncture on the outcomes of IVF-ET (Stener-Victorin et al., 1999). These studies had variable designs and generally yielded inconclusive or conflicting results, rendering the clinical decision of whether to recommend or not the use of acupuncture during IVF difficult.

We conducted a systematic review and meta-analysis of randomised controlled trials to determine whether acupuncture performed at the time of ET improves the outcome among women undergoing IVF ET.

\section{METHODS}

\section{Identification of studies}

We searched the computerised databases Medline and Embase from January 1990 to June 2017. We used the following terms as free text terms and MeSH terms (shown in italics): (acupuncture; acupuncture therapy) and (reproductive techniques, assisted; fertilization in vitro; embryo transfer). We also searched the list of references of relevant publications.

\section{Selection criteria, data extraction and quality assessment}

We selected randomised controlled trials that compared acupuncture with sham acupuncture or no treatment. Because we were evaluating acupuncture as a complement to embryo transfer, we considered only trials in which acupuncture was administered within one day of the procedure, with the objective of improving success rates. 
Trials that included intracytoplasmic injection of sperm as well as in vitro fertilisation were eligible. We excluded trials that evaluated other interventions in conjunction with acupuncture.

For trials to be eligible, we had to be able to extract data on at least one of the following outcomes, as recommended: clinical pregnancy (that is, presence of at least one gestational sac or fetal heartbeat, confirmed by transvaginal ultrasound), ongoing pregnancy (that is, pregnancy beyond 12 weeks of gestation, as confirmed by fetal heart activity on ultrasound), or live birth. We included only trials in which acupuncture involved the insertion of needles into traditional meridian points. The needles could be inserted into tender points in addition to the traditional meridian points.

We excluded trial with electrical stimulation of the needles. We also excluded trials of laser acupuncture and electro-acupuncture without needle insertion, because most authorities believe acupuncture involves needle insertion (Smith et al., 2012).

We imposed no restrictions on publication type (that is, either full article or abstract), and restricted the language to English. Two authors (JPC and JES) independently selected articles and extracted data, with disagreements resolved by discussion.

We evaluated the methodological quality of the trials using the Cochrane risk of bias tool. The items evaluated were: concealment of allocation of randomisation sequence (selection bias), allocation concealment (selection bias), blinding of participants and personnel (detection bias), incomplete outcome data (attrition bias), selective reporting (reporting bias) and other biases (Higgins et al., 2011).

\section{Data synthesis and analysis}

The measure of treatment effect was the pooled odds ratio of achieving a clinical pregnancy, ongoing pregnancy, or live birth for women in the acupuncture group compared with women in the control group. For pooled data, summary test statistics were calculated using the Mantel-Haenszel method, via the Rev-Man software, version 5.1 .

Our meta-analyses were based on the number of women randomised (rather than on the number of treatment attempts-that is, cycles of in vitro fertilisation) with the intention-to-treat approach analysis.

\section{Subgroup analysis}

We evaluated heterogeneity using the $\mathrm{I}^{2}$ test (Higgins et al., 2003), which indicates the proportion of variability across trials not explained by chance alone, and the $p$-value of the $\mathrm{X}^{2}$ test of heterogeneity. Although interpreting the importance of inconsistency depends on other factors in addition to the $\mathrm{I}^{2}$ values (e.g. $p$-value from $X^{2}$ test, magnitude and direction of effects), the Cochrane Handbook suggests the following rough guide to interpreting the $\mathrm{I}^{2}$ values: low, moderate, and high to $I^{2}$ values of $25 \%, 50 \%$, and $75 \%$, respectively (Higgins et al., 2003).

If the overall $\mathrm{I}^{2}$ value for all trials was reduced when we separated the trials into subgroups according to source of bias, we would use the subgroup results as primary. Otherwise, the pooled results from all trials would be used for our primary analysis, but with the results from the two subgroups also present.

\section{RESULTS}

Figure 1 shows details of the selection process. Six randomized controlled trials, with 2,376 participants, met the selection criteria. All trials were published in English since 2009, and conducted in three different Western countries (Paulus et al., 2002; Westergaard et al., 2006; Domar et al., 2009; Andersen et al., 2010; Moy et al., 2011) and China (So et al., 2009). They were all published as full reports.

All six trials used a pragmatic design, including typical clinical populations and using typical interventions before and after randomization. All included a broad selection of women undergoing in vitro fertilisation, with a wide range of ages, diagnostic categories of infertility, durations of infertility, and numbers of previous treatment cycles.

The timing of the acupuncture sessions relative to embryo transfer differed somewhat among the trials (Table 1 ). However, in all the trials the women received acupuncture immediately before or immediately after the embryo transfer.

In all trials, the acupuncture protocol and selection of acupuncture points were designed for the sole purpose of improving pregnancy rates.

\section{Risk of bias of included studies}

The trials generally had high internal validity, in terms of randomisation procedures and follow-up of participants. In all trials, the investigators confirmed no losses to follow-up, which is usual for in vitro fertilisation cycles (Figures 2 and 3 ).

Four trials used sham acupuncture controls (Paulus et al., 2002; So et al., 2009; Andersen et al., 2010; Moy et al., 2011); one trial used needles that penetrated the skin at acupuncture points selected not to influence fertility (Andersen et al., 2010), and three used non-penetrating sham needles (Paulus et al., 2002; So et al., 2009; Moy et al., 2011). In all trials, the acupuncture sessions lasted 25-30 minutes (Table 1 ).

In all trials, there were no significant differences between the groups in the mean numbers of embryos transferred, mean age of the women undergoing the procedure, diagnose and use of ICSI. Funnel plot analyses showed that there were nonpublication biases (Figure 4).

\section{Efficacy analysis}

The heterogeneity among studies was high, $\mathrm{I}^{2}$ 69\% $p$-value $=0.006$; therefore, we restricted to those four randomised trials with less than $50 \%$ bias risk (Domar \& Alper, 2013). Acupuncture performed the day of ET was associated with a reduced risk of clinical pregnancy $(0.87,95 \%$ confidence interval 0.77 to 0.98$)$, (Figure 5). The pooled rate difference was $-0.06(-0.12$ to -0.01$)$ for clinical pregnancy.

None of the trials reported significant adverse effects of acupuncture.

\section{DISCUSSION}

After the meta-analysis of the studies with a low-risk of bias, we found that acupuncture performed on the day of ET decreases significantly the risk of achieving a pregnancy. Probably, the main strength of our study is that we randomised controlled trials with low risk of 


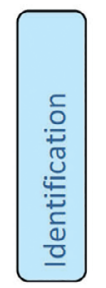

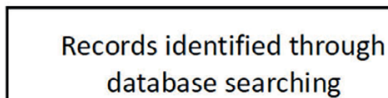

database searching $(n=83)$

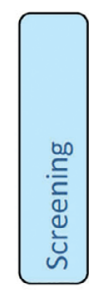

Records after duplicates removed

$$
(n=83)
$$
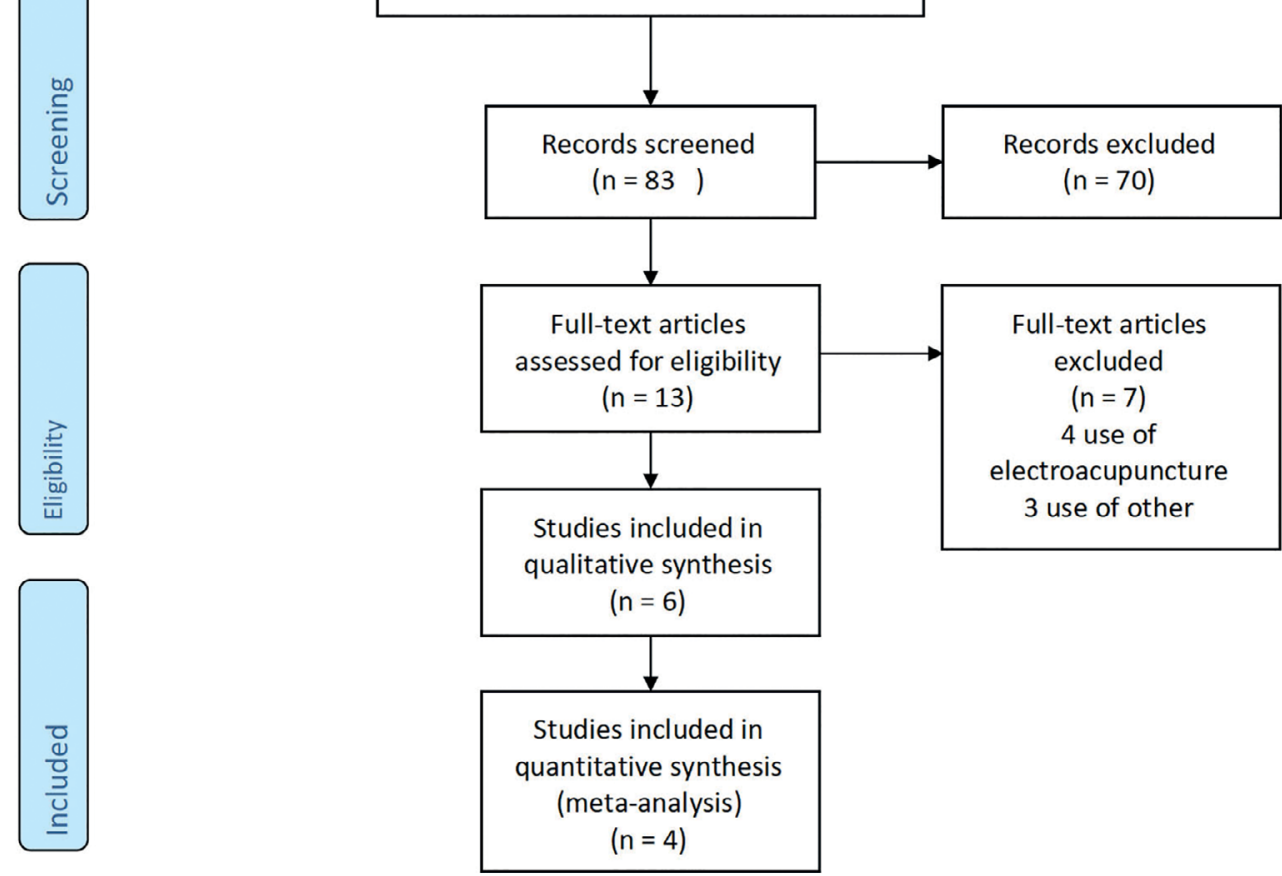

Additional records identified through other sources $(n=0)$

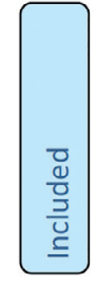

Figure 1. Flow chart of the study selection process for eligible studies in the systematic review

\begin{tabular}{|c|c|c|c|}
\hline Study & Country & Intervention & Results \\
\hline Paulus et al., 2002 & Germany & $\begin{array}{l}80 \text { patients underwent } 25 \text { minutes } \\
\text { of acupuncture before and after ET; } \\
\text { the control were } 80 \text { patients without } \\
\text { acupuncture }\end{array}$ & $\begin{array}{l}34 / 80 \text { got pregnant in the acupuncture } \\
\text { group, versus } 21 / 80 \text { in the control } \\
\text { group }\end{array}$ \\
\hline Westergaard et al., 2006 & Denmark & $\begin{array}{l}95 \text { patients underwent } 25 \text { minutes } \\
\text { of acupuncture before and after ET; } \\
\text { the control were } 87 \text { patients without } \\
\text { acupuncture }\end{array}$ & $\begin{array}{l}40 / 95 \text { got pregnant in the acupuncture } \\
\text { group, versus } 24 / 87 \text { in the control } \\
\text { group }\end{array}$ \\
\hline Domar et al., 2009 & USA & $\begin{array}{l}78 \text { patients underwent } 25 \text { minutes } \\
\text { of acupuncture before and after ET; } \\
\text { the controls were } 68 \text { patients without } \\
\text { acupuncture }\end{array}$ & $\begin{array}{l}39 / 78 \text { got pregnant in the acupuncture } \\
\text { group, versus } 29 / 68 \text { in the control } \\
\text { group }\end{array}$ \\
\hline So et al., 2009 & China & $\begin{array}{l}185 \text { patients underwent } 25 \text { minutes of } \\
\text { acupuncture before and after ET; the } \\
\text { controls were } 185 \text { patients with sham } \\
\text { acupuncture }\end{array}$ & $\begin{array}{l}81 / 185 \text { got pregnant in the } \\
\text { acupuncture group, versus } 102 / 185 \text { in } \\
\text { the control group }\end{array}$ \\
\hline Andersen et al., 2010 & USA & $\begin{array}{l}314 \text { patients underwent } 30 \text { minutes of } \\
\text { acupuncture before and after ET; the } \\
\text { control were } 321 \text { patients with sham } \\
\text { acupuncture }\end{array}$ & $\begin{array}{l}126 / 314 \text { got pregnant in the } \\
\text { acupuncture group, versus } 149 / 321 \text { in } \\
\text { the control group }\end{array}$ \\
\hline Moy et al., 2011 & USA & $\begin{array}{l}86 \text { patients underwent } 25 \text { minutes of } \\
\text { acupuncture before and after ET; the } \\
\text { controls were } 74 \text { patients with sham } \\
\text { acupuncture }\end{array}$ & $\begin{array}{l}39 / 86 \text { got pregnant in the acupuncture } \\
\text { group, versus } 39 / 74 \text { in the control } \\
\text { group }\end{array}$ \\
\hline
\end{tabular}




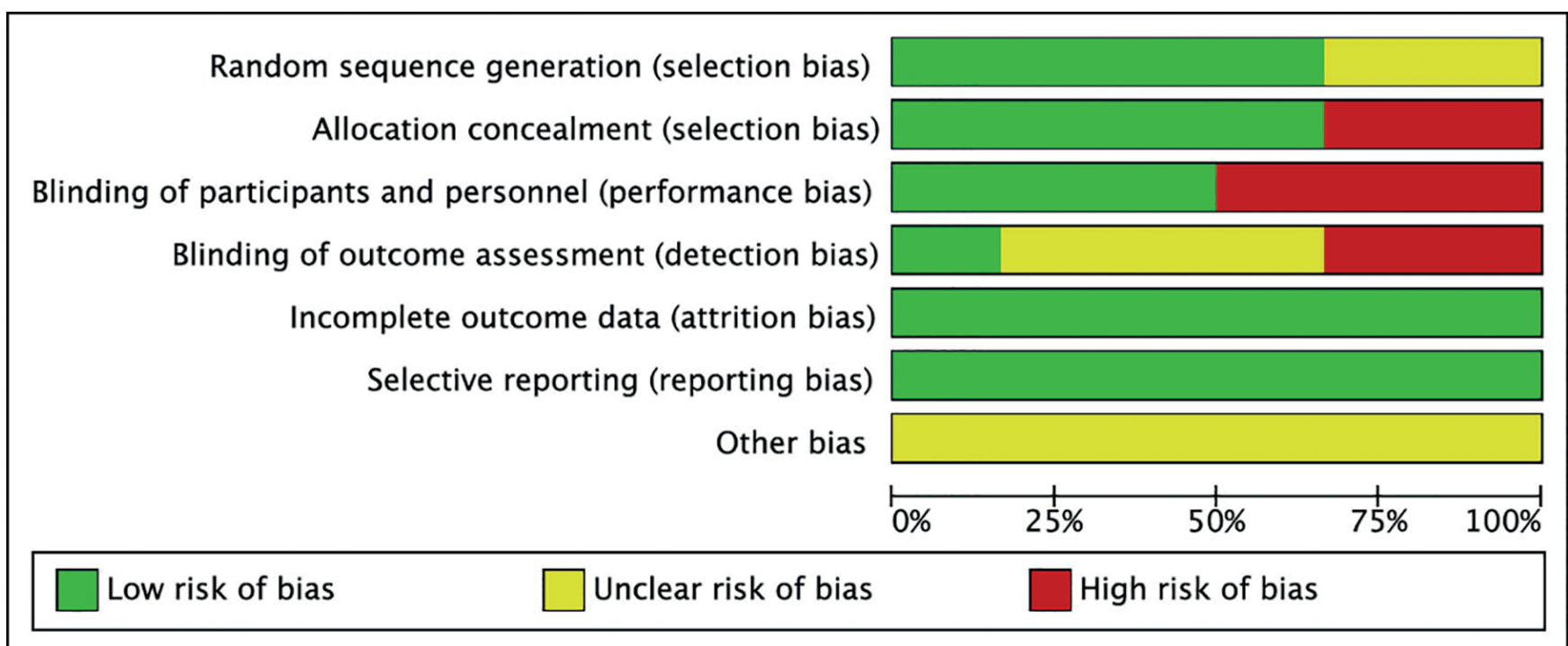

Figure 2. Risk of bias graph

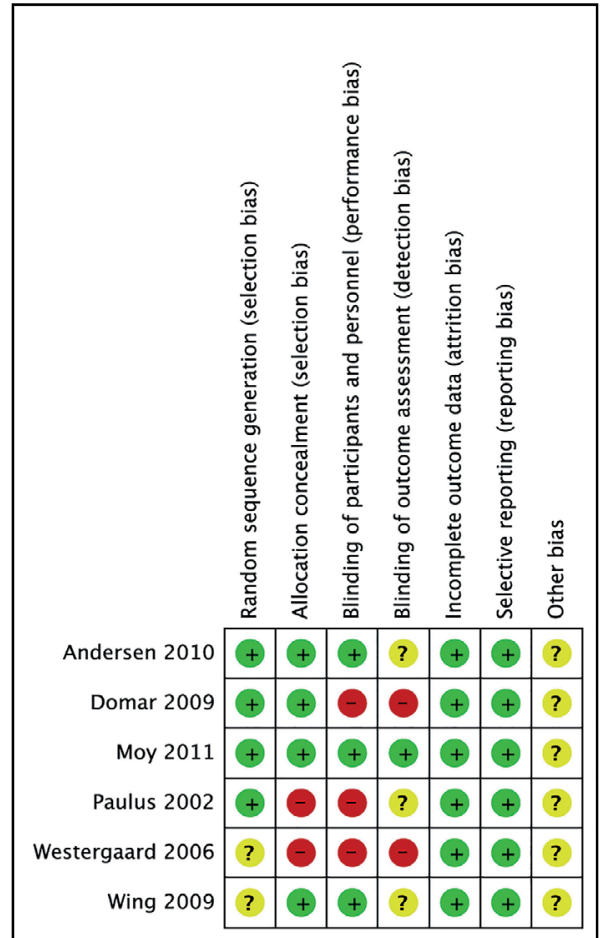

Figure 3. Risk of bias summary

bias, and low heterogeneity. Nevertheless, the main weakness is that there are few studies dealing with acupuncture at the time of ET, and we could not find any study published after 2011.

Other studies have dealt with these questions (Qian et al., 2017; Manheimer et al., 2008; 2013). Qian et al. (2017) ran a meta-analysis involving 30 studies, including 6,344 women, and found an improvement in the OR of clinical pregnancy of 1.26 ( $95 \%$ confidence interval,
1.06-1.50). However, they compared any accepted form of acupuncture, and did not evaluate the risk of bias among the studies. Manheimer et al. (2008) published a meta-analysis of seven trials, including 1,366 women, and found a significant improvement in the OR of pregnancy of 1.5 (95\% confidence interval 1.27-2.14); however, they analysed studies with or without any adjuvant therapy, thus adding a source of bias to the study. Later, in 2013, the same group published a new meta-analysis of 16 trials, with a total of 4,021 women, and found no difference in the OR of pregnancy $(1.65 ; 95 \%$ confidence interval 0.96-1.31), again the use of any adjuvant therapy was not a cause of exclusion (Manheimer et al., 2013).

According to our findings, physicians should encourage their patients to avoid undergoing acupuncture on the day of ET, since it diminishes the chances of getting pregnant. It still has to be elucidated the mechanisms by which acupuncture negatively affects embryo implantation. So far, no evidence of a significant effect of acupuncture on vascular biomarkers and well-being (Phy et al., 2017), endometrial and subendometrial vascularity, serum cortisol (So et al., 2009)concentration and anxiety level has been demonstrated.

In conclusion, acupuncture performed on the day of ET has a significant effect on embryo implantation, however, a negative one. Therefore, clinicians should encourage their patients to avoid this technique the day of ET.

\section{CONFLICT OF INTEREST}

The authors declare no potential conflict of interest.

\section{Corresponding Author:}

Juan Enrique Schwarze

Reproductive Medicine Unit, Clinica Las Condes

Santiago, Chile

E-mail: jschwarze@clinicalascondes.cl;

jeschwarze@gmail.com 


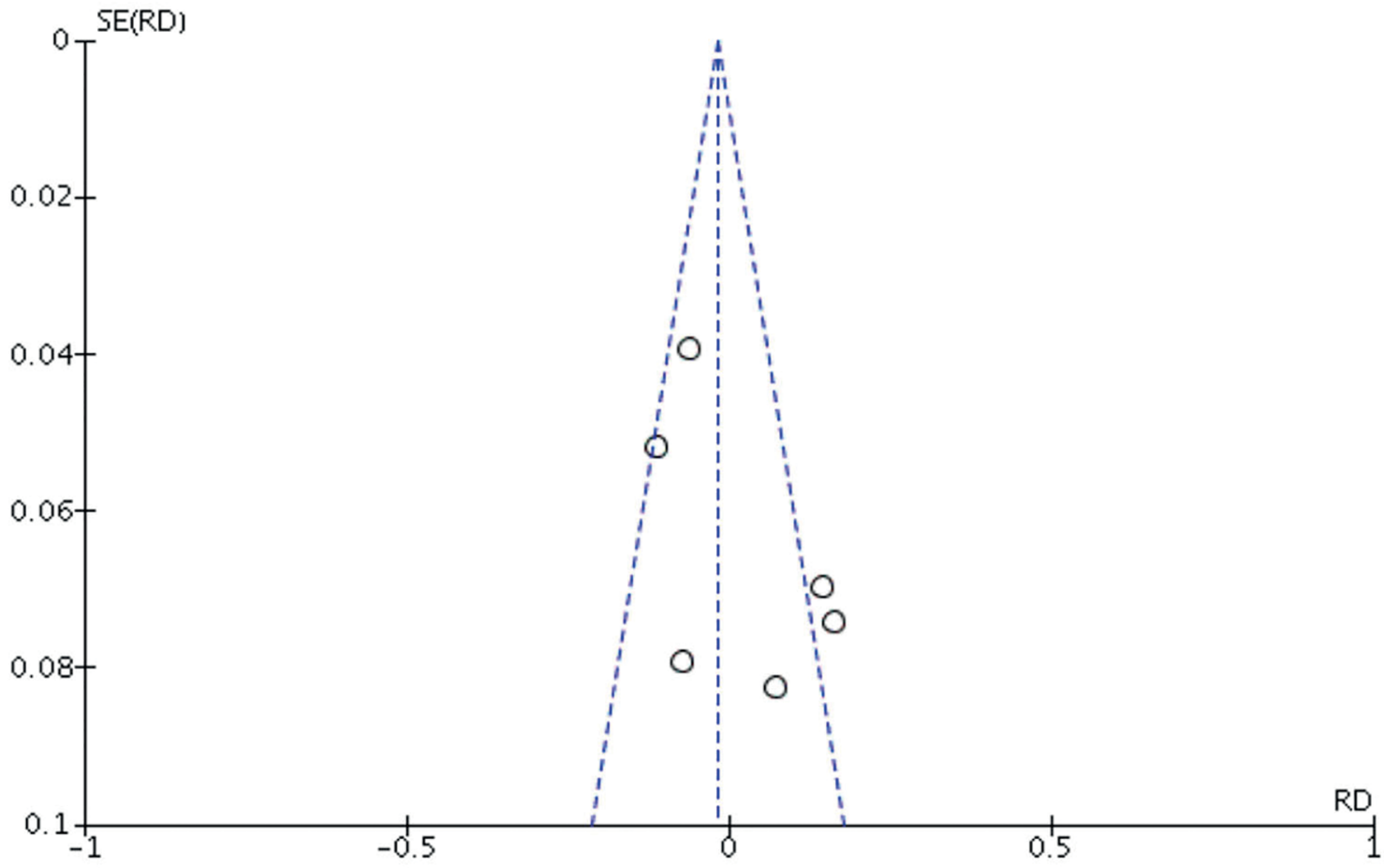

Subgroups

O All studies

Figure 4. Funnel plot analysis

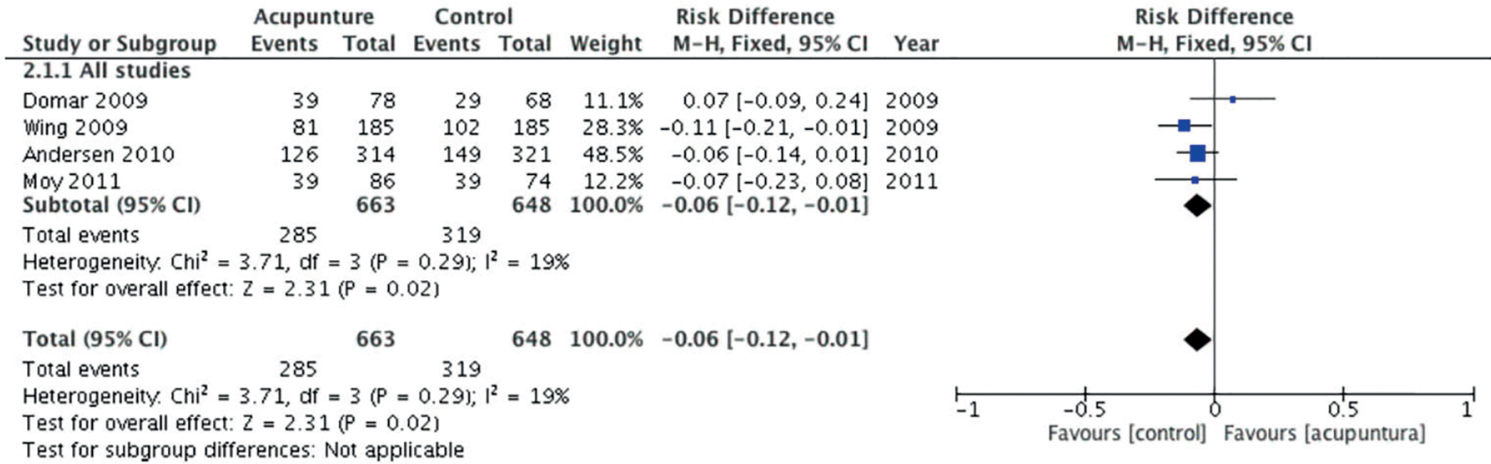

Figure 5. Forest plot of comparison: Acupuncture versus control, outcome: Clinical pregnancy per embryo transfer

\section{REFERENCES}

Andersen D, Løssl K, Nyboe Andersen A, Fürbringer J, Bach $\mathrm{H}$, Simonsen J, Larsen EC. Acupuncture on the day of embryo transfer: a randomized controlled trial of 635 patients. Reprod Biomed Online. 2010;21:366-72. PMID: 20638338 DOI: $10.1016 /$ j.rbmo.2010.03.029

Chang $\mathrm{R}$, Chung PH, Rosenwaks $Z$. Role of acupuncture in the treatment of female infertility. Fertil Steril. 2002;78:1149-53. PMID: 12477502DOI: 10.1016/S00150282(02)04348-0
Domar AD, Meshay I, Kelliher J, Alper M, Powers RD. The impact of acupuncture on in vitro fertilization outcome. Fertil Steril. 2009;91:723-6. PMID: 18314118 DOI: $10.1016 /$ j.fertnstert.2008.01.018

Domar AD, Alper MM. Acupuncture - placebo? Fertil Steril. 2013;100:e21. PMID: 23932818 DOI: 10.1016/j.fertnstert.2013.07.1970

Higgins JP, Thompson SG, Deeks J], Altman DG. Measuring inconsistency in meta-analyses. BMJ. 2003;327:557-60. PMID: 12958120 DOI: $10.1136 / \mathrm{bmj} .327 .7414 .557$ 
Higgins JP, Altman DG, Gøtzsche PC, Jüni P, Moher D, Oxman AD, Savovic J, Schulz KF, Weeks L, Sterne JA; Cochrane Bias Methods Group; Cochrane Statistical Methods Group. The Cochrane Collaboration's tool for assessing risk of bias in randomised trials. BMJ. 2011;343:d5928. PMID: 22008217 DOI: $10.1136 / \mathrm{bmj} . \mathrm{d} 5928$

Manheimer E, Zhang G, Udoff L, Haramati A, Langenberg $P$, Berman BM, Bouter LM. Effects of acupuncture on rates of pregnancy and live birth among women undergoing in vitro fertilisation: systematic review and meta-analysis. BMJ. 2008;336:545-9. PMID: 18258932 DOI: 10.1136/bmj.39471.430451.BE

Manheimer $E$, van der Windt $D$, Cheng $K$, Stafford $K$, Liu J, Tierney J, Lao L, Berman BM, Langenberg P, Bouter LM. The effects of acupuncture on rates of clinical pregnancy among women undergoing in vitro fertilization: a systematic review and meta-analysis. Hum Reprod Update. 2013;19:696-713. PMID: 23814102 DOI: 10.1093/humupd/dmt026

Moy I, Milad MP, Barnes R, Confino E, Kazer RR, Zhang $X$. Randomized controlled trial: effects of acupuncturex on pregnancy rates in women undergoing in vitro fertilization. Fertil Steril. 2011;95:583-7. PMID: 20646688 DOI: 10.1016/j.fertnstert.2010.05.024

Phy JL, Orlando J, Zhang Y, Garos S, Penrose L, Prien S, Huang J. Effect of acupuncture on vascular biomarkers and psychological well-being of women undergoing IVF. Fertil Steril. 2017;108:e302-3. DOI: 10.1016/j.fertnstert.2017.07.894

Qian Y, Xia XR, Ochin H, Huang C, Gao C, Gao L, Cui YG, Liu JY, Meng Y. Therapeutic effect of acupuncture on the outcomes of in vitro fertilization: a systematic review and meta-analysis. Arch Gynecol Obstet. 2017;295:543-58. PMID: 27995371 DOI: 10.1007/s00404-016-4255-y

Smith CA, Grant S, Lyttleton J, Cochrane S. Using a Delphi consensus process to develop an acupuncture treatment protocol by consensus for women undergoing Assisted Reproductive Technology (ART) treatment. BMC Complement Altern Med. 2012;12:88. PMID: 22769059 DOI: $10.1186 / 1472-6882-12-88$
So EW, Ng EH, Wong YY, Lau EY, Yeung WS, Ho PC. A randomized double blind comparison of real and placebo acupuncture in IVF treatment. Hum Reprod. 2009;24:341-8. PMID: 18940896 DOI: 10.1093/humrep/den380

Stener-Victorin E, Waldenström U, Andersson SA, Wikland $M$. Reduction of blood flow impedance in the uterine arteries of infertile women with electro-acupuncture. Hum Reprod. 1996;11:1314-7. PMID: 8671446 DOI: 10.1093/oxfordjournals.humrep.a019378

Stener-Victorin E, Waldenström $U$, Nilsson L, Wikland $M$, Janson PO. A prospective randomized study of electro-acupuncture versus alfentanil as anaesthesia during oocyte aspiration in in-vitro fertilization. Hum Reprod. 1999; 14:24804. PMID: 10527973 DOI: 10.1093/humrep/14.10.2480

Westergaard LG, Mao Q, Krogslund M, Sandrini S, Lenz $\mathrm{S}$, Grinsted J. Acupuncture on the day of embryo transfer significantly improves the reproductive outcome in infertile women: a prospective, randomized trial. Fertil Steril. 2006;85:1341-6. PMID: 16600232 DOI: $10.1016 /$ j.fertnstert.2005.08.070

Yang $\mathrm{CH}$, Lee $\mathrm{BH}$, Sohn $\mathrm{SH}$. A possible mechanism underlying the effectiveness of acupuncture in the treatment of drug addiction. Evid Based Complement Alternat Med. 2008;5:257-66. PMID: 18830420 DOI: $10.1093 /$ ecam/nem081

Zegers-Hochschild F, Schwarze JE, Crosby JA, Musri C, Urbina MT; Latin American Network of Assisted Reproduction (REDLARA). Assisted reproductive techniques in Latin America: the Latin American Registry, 2013. Reprod Biomed Online. 2016a;32:614-25. PMID: 26997476 DOI: 10.1016/j.rbmo.2016.02.012

Zegers-Hochschild F, Schwarze JE, Crosby JA, Musri C, Urbina MT; Latin American Network of Assisted Reproduction (REDLARA). Assisted reproductive techniques in Latin America: The Latin American Registry, 2013. JBRA Assist Reprod. 2016b;20:49-58. PMID: 26997476 DOI: 10.1016/j.rbmo.2016.02.012 